STRUCTURAL SCIENCE CRYSTAL ENGINEERING MATERIALS

ISSN 2052-5206

\section{Industrial Inorganic Chemistry. By Mark Anthony Benvenuto. De Gruyter, 2015. Paperback, xiii + 197 pages. ISBN 978-3-11-033032-8. Price EUR 69,95, USD 98.00, GBP 52.99}

\author{
Patrice Berthod*
}

Université de Lorraine, Institut Jean Lamour, UMR 7198, Vandoeuvre-les-Nancy, F-54506, France, and CNRS, Institut Jean Lamour, UMR 7198, Vandoeuvre-les-Nancy F-54506, France. *Correspondence e-mail: patrice.berthod@univ-lorraine.fr

Keywords: book review; industrial inorganic compounds.

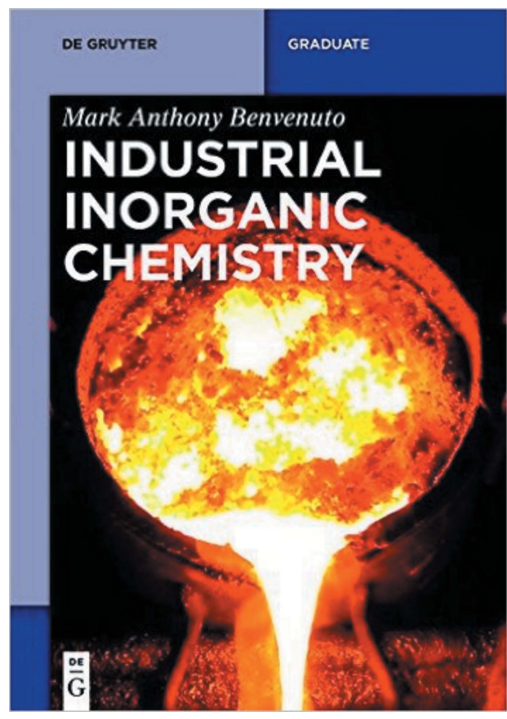

(C) 2016 International Union of Crystallography
This book, which collects much data at several levels - history, extraction, treatment, production, economy, uses, recycling - for a great number of industrial inorganic substances, starts with a short 2-page introduction, presented as Chapter 1, which includes the significance of distinction of materials as a function of their origin.

The first substance of interest, sulfuric acid, is the subject of Chapter 2. Sulfur, its oxides, and the different compounds in which it is present, are listed with selected data attached (history, uses, chemical reactions...). Chapter 3 considers several industrial gases (oxygen, nitrogen, hydrogen, rare gases...), and different inorganic compounds involving nitrogen are discussed in Chapter 4, with the same type of data as previously mentioned.

Fertilizers based on nitrogen, phosphorus and potassium are thereafter discussed in Chapter 5, with particular emphasis on geographic information. Chapters 6 and 7 concern calcium and sodium, respectively, and the compounds involving each of them. Note in the body of Chapter 7, which is devoted to sodium, the curious presence of a paragraph dealing with titanium oxide. This insertion is obviously an error and should be moved to Chapter 14.

The case of materials involving fluorine and its compounds (acids, Teflon ... ) is treated in Chapter 8, while Chapter 9 deals with some substances and materials which are difficult to classify as inorganic or organic (such as carbon black, borax or asphalt). The case of water and its treatments is addressed in Chapter 10.

Metals are treated in the following chapters: Chapter 11 for iron and steels (why not cast irons?), Chapter 12 for aluminium and its alloys, Chapter 13 for copper and associated alloys (brass and bronze notably), Chapter 14 for titanium (it is here that the infamous paragraph is lacking), chromium, mercury, gold, silver, lead, tin, the platinum group metals, technetium, tantalum and niobium. Chapters 11-13 are the most substantial in terms of history; the corresponding ore, the industrial techniques of production and refinement, geographic and economic data, the alloys based on the element of interest, and the recycling are all reviewed.

Chapters 15 and 16 address the case of the rare or rare-earth elements (e.g. yttrium) and the case of radioactive elements (uranium, thorium). Chapters 17 and 18 concern silicon, and metals and alloys with low density (aluminium, magnesium, titanium, beryllium), aerogels of various types, zeolites, fullerenes, with the same points of interest as before (production, use, recycling). In the two following Chapters (19 and 20) ceramics and hard materials (diamond, nitrides of silicon, boron and carbon, metal borides) are discussed.

At the end is only an index, curiously without any general conclusions.

Thus this book is interesting, easy to read and illustrated by the presence of tables, graphs, schemes and photographs. A lot of inorganic materials are listed, with short texts and accompanying data of various nature: geographic, economic, schemes of processes, chemical reaction equations and so on (but the information provided is unfortunately often of a different nature material by material, e.g. only geographic, economic ... for one of the materials and only fabrication processes, chemical reactions ... for another). However, with only 200 pages of small size with large spaces between lines, and then a 
rather short total text illustrated by a few figures and photographs regarding the great quantity of materials and substances, the part addressing a particular material or substance is too short and therefore incomplete. Consequently, the book logically looks like a catalog in which one cannot really exploit the given information for many of the addressed materials and substances. The interest above all is the discovery of the great variety of existing inorganics, before reading a little more specialized books to start collecting information in enough quantity to be really useful. 